\title{
Serie tv di culto, ovvero il culto delle serie tv Protagonisti seriali come cult icons nell'epoca della complex tv
}

\author{
Andrea Bernardelli \\ Università degli Studi di Perugia \\ andrea.bernardelli@unipg.it \\ $<$ http://unipg.academia.edu/AndreaBernardelli >
}

\begin{abstract}
Last generation tv series - the tv series of the last two decades - seem to be a true obsession for their audiences, to the point these series can be defined as cult series. The main element which raises such veneration is the protagonist's figure, or of a character who becomes a cult icon, and the mechanisms that make this possible. So the question is whether a good marketing campaign suffices to create the worship of a tv series, or if on the contrary, we should also work on building up a protagonist that could become a cult icon for the audience. Can we determine which narrative strategies can induce this process? And how can a cult character be built in television series?
\end{abstract}

\section{Keywords}

Tv series; Fictional character; Media cult; Narratology

\section{Sommario/Content}

1. Premessa

2. Mito e personaggio in Eco

3. Il culto nella narrazione audiovisiva

4. Il culto del personaggio "difficile"

Bibliografia 


\section{Ocula}

Vol 21, No 22 (April 2020) • DOI: 10.12977/ocula2020-16

Andrea Bernardelli | Serie tv di culto, ovvero il culto delle serie tv. Protagonisti seriali come cult icons nell'epoca della complex tv

\section{Premessa}

Negli ultimi decenni alcune serie televisive hanno assunto un ruolo culturale sempre più rilevante, fino a poterle definire serie di culto (Monteleone 2005, Scaglioni 2006, Maio 2013, Mittell 2015). In sostanza quello che accade è che si formano delle più o meno ristrette comunità di spettatori che trovano in quel prodotto narrativo un punto di riferimento di discussione culturale, costituendo in tal modo un fandom (v. ad es. Hills 2002, Gray et al. 2007; Duffett 2013, Williams 2015). Questo è testimoniato, oltre che dall'interesse degli accademici per il fenomeno, dal numero dei siti dedicati al fandom, vale a dire alla discussione e spesso anche alla riscrittura - la fanfiction (v. Calabrese e Conti 2019) - di serie tv. Ma cosa si intende nello specifico per serie di culto? E attraverso quale meccanismo si crea il culto di una serie tv? Perché solo alcune serie tv diventano di culto e altre no?

In sostanza in tutti questi casi è centrale il fatto che nella cultura contemporanea circolino, ma come d'altronde accadeva in passato, narrazioni in senso esteso che vengono riconosciute come vere e proprie mitologie contemporanee, insiemi di racconti transmediali che fanno sistema e che vengono a costituire un elemento di identità culturale (Ortoleva 2019). L'icona culturale, genericamente intesa, può essere vista come il risultato della catacresizzazione, del "congelamento" o fissazione, di un singolo aspetto di un racconto culturalmente, o 'mitologicamente', rilevante. Un luogo, un oggetto, una persona diventano l'elemento di richiamo cognitivo dell'intero discorso mitologico in senso lato. Un personaggio diventa icona culturale quando viene estrapolato e isolato dalla narrazione mitologica, come è accaduto per la figura di Ulisse e per le sue diverse migrazioni intertestuali e intermediali (v. ad es. Boitani 1992). In questi casi il personaggio rimanda per antonomasia a specifici valori culturali e identitari, soggetti a forme di definizione e ricontrattazione storica e contestuale, esemplificati dalla serie dei racconti che formano il corpus della sua narrazione estesa.

Tornando all'oggetto specifico del nostro discorso, nelle serie televisive di culto degli ultimi decenni ci troviamo spesso di fronte alla centralità della figura di un protagonista o di uno specifico personaggio (nelle serie corali). Questo personaggio in alcuni casi diventa la cult icon di una comunità di fans di quella specifica serie tv. Quindi l'essere di culto della serie tv sembra essere mediato dalla costruzione della figura di un personaggio che per antonomasia diventa la cult icon di quel prodotto narrativo.

La costruzione del personaggio, e in particolare della figura del protagonista, in una narrazione seriale è uno degli aspetti fondamentali per dare continuità e coerenza al mondo narrativo rappresentato. $\mathrm{E}$ di conseguenza non può essere estraneo alla costruzione del culto per quella narrazione. Basti pensare a quali sono gli esempi di cult series riportati, ad esempio, da Monteleone (2005): serie in cui già dal titolo è evidente la centralità del, o della, protagonista, come Buffy l'ammazzavampiri, o Ally McBeal, e anche serie in cui questo tratto non è evidente fin da principio, ma in cui è fondamentale la centralità di una specifica figura o coppia di personaggi, dai Mulder e Scully di X-Files, al dottor Doug Ross (George Clooney) di ER. 


\section{Ocula}

Vol 21, No 22 (April 2020) • DOI: 10.12977/ocula2020-16

Andrea Bernardelli | Serie tv di culto, ovvero il culto delle serie tv. Protagonisti seriali come cult icons nell'epoca della complex tv

\section{Mito e personaggio in Eco}

Ma cosa può dire la semiotica a riguardo? Del meccanismo che lega il culto di un testo alla particolare costruzione della figura del personaggio possiamo trovare una prima forma di analisi semiotica collegando tra loro alcuni saggi di Eco. L'autore si occupa delle forme del mito nel mondo contemporaneo nel saggio "Il mito di Superman" (Eco 1964). Secondo Eco storicamente la mitizzazione avveniva per via istituzionale, dall'alto verso il basso. Così per il culto dei santi era l'istituzione religiosa che ne definiva i termini e i confini. Ma questo processo si intrecciava con l'intervento dal basso della sensibilità popolare che collegava il culto dei santi con tradizioni diverse e ne ricostruiva a proprio modo la narrazione. Si trattava dell'intreccio (intertestuale) tra una mitopoiesi diretta e istituzionale e una mitopoiesi spontanea o popolare. Ma con la modernità tutto questo entra in crisi e si rompe il rapporto più o meno prefissato tra le immagini e i significati filosofico-religiosi da esse veicolati. Le arti si trovano così a istituire "simboli soggettivi", creazione personale dell'autore, che solo in parte svolgono il ruolo dei precedenti "simboli oggettivi", come è avvenuto nel caso della poetica del correlativo oggettivo della letteratura modernista (Eco 1964: 221).

$\mathrm{Nel}$ mondo contemporaneo esiste secondo Eco una tendenza a ricostruire su basi popolari quella perduta "universalità di sentire e di vedere". Nella civiltà industriale si crea una comunicazione simbolica che parte da un meccanismo simile a quello della mitopoiesi del poeta moderno, privata e soggettiva, ma che rinvia ad aspirazioni e bisogni più generali e collettivi. Si tratta della creazione di miti contemporanei privata e soggettiva, ma che parte dall'alto, non da istituzioni religiose però, ma dagli uffici marketing delle grandi aziende. L'esempio che usa Eco è quello del comics, contemporaneamente oggetto di una fruizione e risemantizzazione popolare, ma che si basa su un repertorio mitologico che proviene dall'alto, dall'industria editoriale.

Eco trova in tal senso interessante la figura simbolica di Superman. Si tratta apparentemente della reincarnazione del mito dell'eroe dotato di poteri superiori a quelli dell'uomo comune, ma con una particolare declinazione che lo rende icona contemporanea. L'alieno Superman si nasconde sotto l'identità umana del giornalista Clark Kent, pauroso, timido, goffo, miope, e disprezzato da tutti, in particolare dalla collega Lois Lane, innamorata del suo alter ego Superman. Questa doppia identità del supereroe permette l'identificazione con il lettore medio, carico di ogni complesso di inferiorità, che trova in Superman/Kent la possibilità nascosta di un riscatto superomistico.

Ma il personaggio del comics nasce in un nuovo regime narrativo, quello della civiltà del romanzo. Se il mito portava alla narrazione continua di ciò che era dato, avvenuto una volta per sempre, immutabile e irreversibile (il mito di Ercole) e che finiva solo per narrare in modo ogni volta un po' diverso la stessa storia, dopo l'avvento del romanzo si cerca l'invenzione, la novità, l'imprevedibilità di ciò che sta per accadere nel momento in cui viene raccontato. Così è cambiato lo status del personaggio che è sì punto di riferimento di comportamenti ed emozioni che appartengono a tutti noi - in quanto tale simbolico -, 


\section{Ocula ${ }^{22}$}

Vol 21, No 22 (April 2020) • DOI: 10.12977/ocula2020-16

Andrea Bernardelli | Serie tv di culto, ovvero il culto delle serie tv. Protagonisti seriali come cult icons nell'epoca della complex tv

ma che manca della universalità del personaggio mitico tradizionale che rinviava a una realtà sovrannaturale. Il personaggio del romanzo, e quello del comics, diventano icona universale attraverso la narrazione di una vicenda particolare.

Nel saggio "L'uso pratico del personaggio" Eco aveva contrapposto due diverse caratterizzazioni del personaggio (Eco 1964a). Riprendendo la teoria di Lukács del personaggio, metteva in evidenza come esistano personaggi le cui caratteristiche individuali devono dare espressione a problemi collegati ad una più vasta concezione del mondo - i personaggi tipo -, ma anche personaggi costruiti secondo un diverso modello che lui definiva attraverso il concetto retorico di topos. Nella retorica classica i topoi erano i "luoghi comuni", i concetti accettati convenzionalmente dalla maggioranza all'interno di una cultura, e che il buon retore doveva conoscere per fondare una argomentazione persuasiva. Il personaggio topos è quindi convenzionale e di facile memorizzazione: meno ricco di collegamenti con le problematiche storico-culturali a cui rinvia il personaggio tipico; il personaggio topos ha una maggiore facilità di collegarsi alla memoria del lettore (come per il piatto personaggio di D'Artagnan). Infatti la sola ricchezza del personaggio topico sta proprio nel rinvio al già noto (dal punto di vista dell'autore dal fare riferimento al già fatto). Ma nel discorso sul mito contemporaneo questa contrapposizione permette di identificare il personaggio mitologico classico come topos, mentre il personaggio mitico contemporaneo, figlio del romanzo, è un personaggio come tipo. ${ }^{1}$

Così il personaggio del comics, in quanto "tipico", si trova a dover assolvere al duplice compito di essere un archetipo delle aspirazioni collettive della cultura popolare contemporanea, ma deve anche essere soggetto al meccanismo di sviluppo e di trasformazione della logica romanzesca. Il personaggio di Superman risolve questa contraddizione attraverso un particolare meccanismo narrativo, quello della serialità e un particolare uso della temporalità seriale. Si tratta dell'iterazione di uno schema narrativo tipico della serialità episodica che comporta la situazione paradossale di fare scorrere il tempo nel singolo episodio, ma non nella sequenza degli episodi nel loro insieme. Sistema che però mantiene una particolare forma di coerenza e coesione del mondo narrativo che non si "consuma" nel tempo così come resta immutabile il suo protagonista. La serialità permette al personaggio mitico contemporaneo di essere sempre uguale a se stesso, ma continuamente diverso (vedi anche Eco 1985).

Queste osservazioni si potrebbero poi ricollegare a quanto detto da Eco sulla costruzione del fenomeno del culto nel mondo dei mass media nel saggio "Casablanca, o la rinascita degli dei" (Eco 1977: 138-143). Il film Casablanca

1 La dicotomia personaggio tipo/topos di Eco è stata riutilizzata da Denson e Mayer (2018) per una interessante caratterizzazione dei personaggi seriali. Gli autori distinguono i serial character dalle serial figures, dove i primi sono personaggi tondi, che vengono portati ad un pieno sviluppo nel corso di una singola narrazione seriale, mentre i secondi sono più simili ai personaggi topoi di Eco, piatti e immutabili ogni volta che vengono riutilizzati in una narrazione seriale. Le serial figures sono soggette a trasformazione solo in ragione del loro passaggio da una forma mediale ad un'altra, sono sensibili al medium, alla plurimedialità. Sarebbe proprio la piattezza delle serial figures a permettere la loro trasponibilità e quindi la loro persistenza come icone culturali. 


\section{Ocula}

Vol 21, No 22 (April 2020) • DOI: 10.12977/ocula2020-16

Andrea Bernardelli | Serie tv di culto, ovvero il culto delle serie tv. Protagonisti seriali come cult icons nell'epoca della complex tv

(1942) è secondo Eco diventato oggetto di culto perché contiene tutti gli archetipi possibili. Ci sono gli archetipi culturali e letterari più tradizionali, l'Amore Infelice e Contrastato, la Fuga e il Passaggio alla Terra Promessa, o la Salvezza, l'Amicizia e la Purezza. Ma anche gli archetipi che coinvolgono direttamente i personaggi, attraverso la personificazione dell'Avventuriero Ambiguo e Tormentato (Rick), della Donna Enigmatica e Fatale (Else), e del Cattivo, sia quello senza redenzione, il nazista maggiore Strasser, sia quello che si redime, il capitano Renault. Eco dice che è la forte "risonanza intertestuale" di questi archetipi a rendere memorabile, nel senso proprio di qualcosa che rimane nella memoria, per lo spettatore quel film altrimenti tecnicamente discutibile. Gli stessi attori portano con sé la memoria delle loro precedenti interpretazioni che fanno sì che per lo spettatore i personaggi del film vengano arricchiti da questa ulteriore relazione intertestuale.

Volendo sintetizzare la visione di Eco in merito, le narrazioni mediali contemporanee diventano oggetto di culto grazie all'intervento dei rimandi intertestuali, attivi in particolare sulla figura del personaggio. Ma anche su una particolare forma di costruzione del mito contemporaneo, basato sulla serialità e sulla specifica forma di coesione del mondo narrativo che ne deriva. Le due forme si intrecciano in quella che Eco ha chiamato "l'innovazione nel seriale" (Eco 1985) in cui attraverso l'intertestualità la serialità narrativa perde la sua banale ripetitività arricchendosi nella catena dei rinvii e dei rimandi culturali, portandola così in direzione della sua caratterizzazione come mito contemporaneo.

\section{Il culto nella narrazione audiovisiva}

Una particolare importanza nella cultura contemporanea è rivestita dai fenomeni di culto che riguardano le forme della narrazione audiovisiva, cinema e televisione. Il problema è che l'identificazione di un film o di una serie tv come oggetto di culto da parte di un fandom sembra derivare più dalle scelte degli spettatori che da dati oggettivi del testo. Centrale sembra essere il modo in cui viene recepito un particolare prodotto culturale, e come una parte del pubblico ne selezioni alcuni aspetti ritenuti significativi, ritenendoli marcati o iconici, in modo apparentemente idiosincratico.

In ambito cinematografico il culto sembra essere legato in primo luogo alle sottoculture, alla pratica dei midnight movies, al gusto del kitsch e dell'estremo, a scelte che vanno in direzione di prodotti fuori dalle logiche commerciali mainstream (v. Maio 2013: 14-15). D'altro canto esiste anche un'altra possibilità di costruzione di un culto cinematografico che è quella invece in parte derivata dalle stesse logiche divistiche e commerciali hollywoodiane. Film di culto sono quelli legati al nome di un celebre attore, possibilmente dotato di una fama oscura o "maledetta", da James Dean a John Belushi, oppure a grandi registi che hanno segnato la storia del cinema attraverso il loro particolare stile espressivo, da Hitchcock a Kubrick. È evidente come sia estremamente difficile creare un film di culto attraverso strategie di marketing, vale a dire dall'alto, in modo istituzionale. Ma, come sottolinea Barbara Maio, esistono due vie che sembrano condurre alla possibilità che un testo audiovisivo possa 


\section{Ocula}

Vol 21, No 22 (April 2020) • DOI: 10.12977/ocula2020-16

Andrea Bernardelli | Serie tv di culto, ovvero il culto delle serie tv. Protagonisti seriali come cult icons nell'epoca della complex tv

diventare oggetto di culto, la prima è la presenza di una figura autoriale forte e riconosciuta, la seconda è la costruzione di una particolare tipologia di personaggio protagonista, articolato e complesso. Ma in che modo, nello specifico delle serie tv, questi due aspetti possono concorrere alla costruzione del cult?

Matt Hills (2002), cercando di identificare gli elementi che caratterizzano in generale le fan cultures, si trova a un certo punto di fronte alla questione specifica di definire il modo in cui alcune narrazioni all'interno di specifiche comunità interpretative raggiungano lo status di testi di culto (cult texts) (Hills 2002: 131). Secondo Hills la definizione del corpus dei testi oggetto di culto da parte dei fans avviene secondo due principali meccanismi, uno extratestuale e l'altro testuale. Nel primo caso entrano in gioco processi di identificazione e di valorizzazione derivanti dalle dinamiche di audience, vale a dire del pubblico e del suo giudizio. Si tratta di meccanismi potenzialmente oggetto di analisi di tipo sociologico, antropologico, o psicologico. L'altra metà del meccanismo di creazione del testo di culto è invece in senso lato intratestuale, derivato dalle caratteristiche strutturali e narrative del testo, e allo stesso tempo dai suoi meccanismi produttivi e distributivi. In sostanza come dice Hills:

The cult object is hence neither textually programmable nor entirely textually arbitrary. The media cult is paradoxical [...] in that it is both found (consisting of textual qualities and properties) and 'created' [...] by the viewer. (Hills 2002: 131)

Analizzando quelle che ritiene siano le caratteristiche ricorrenti che uno spettatore identifica come centrali per poter definire un testo come oggetto di culto, le riassume in tre punti principali:

1. l'autorialità (autorism);

2. l'apertura indefinita della narrazione (endlessly deferred narrative);

3. la costruzione di diegesi estese e complesse (hyperdiegesis).

Vediamoli ora in sequenza aggiungendo alle tesi di Hills osservazioni su fenomeni mediali più recenti, o da lui non presi in considerazione.

\subsection{La figura autoriale}

Il primo di questi meccanismi creatori di culto, l'autorialità ("autorism”) è ben noto in ambito cinematografico, vedi il caso del ruolo svolto dalla figura di George Lucas per la saga di Star Wars. Si tratta sempre di creatori ritenuti particolarmente innovativi e geniali la cui presenza, in una produzione cinematografica, genera una immediata attesa nel pubblico. In questi casi la figura autoriale diventa l'elemento di continuità per il media cult, l'etichetta che tiene insieme la produzione legata a quel nome.

Per le serie tv è più difficile identificare una figura creativa unica e centrale come avviene nel cinema, in cui spesso viene identifica nel regista o più raramente nello sceneggiatore (nonostante anche per il cinema si tratti di un lavoro di produzione collettivo). Ma a dispetto della natura collaborativa, e non continuativa (i registi cambiano da episodio ad episodio, gli sceneggiatori a volte di stagione in stagione) del prodotto seriale televisivo, in alcuni casi vie- 


\section{Ocula}

Vol 21, No 22 (April 2020) • DOI: 10.12977/ocula2020-16

Andrea Bernardelli | Serie tv di culto, ovvero il culto delle serie tv. Protagonisti seriali come cult icons nell'epoca della complex tv

ne comunque ad essere identificata una figura autoriale unica (v. Checcaglini 2013). Esistono nell'ambito della serialità televisiva alcuni nomi "di culto", registi, sceneggiatori, produttori, a volte tutt'e tre le cose insieme, che vengono identificati come figure autoriali centrali, come nel caso di Chris Carter (The X-Files, 1993-2018), di Gene Roddenberry (Star Trek, 1966-69), Joss Whedon (Buffy. The Vampire Slayer, 1997-2003; Angel, 1999-2004), Jeffrey J. Abrams (Alias, 2001-6; Lost, 2004-10), Jonathan Nolan (Westworld, 2016-in produzione). Alcune di queste figure sono in realtà celebri anche nell'ambito delle produzioni cinematografiche (Abrams, Nolan), ma anche alcuni registi o sceneggiatori cinematografici già autori di culto in quel settore si sono prestati a lavorare alla produzione di serie televisive, trasmettendo una parte dell'aura del proprio culto già acquisita al prodotto televisivo, come nel caso di David Lynch per Twin Peaks (1990-91), di Jane Campion per Top of the Lake (2013), oppure recentemente di Nicolas Winding Refn con Too Old to Die Young (2019-in produzione).

Forse è per questo motivo, vale a dire la ricerca di una figura autoriale centrale simile a quella del regista in ambito cinematografico, che in molte produzioni statunitensi viene identificato - e utilizzato come una sorta di testimonial di qualità della serie -, la figura dello showrunner. Si tratta spesso di uno sceneggiatore/produttore, a volte anche regista, che svolge il ruolo di responsabile artistico, produttivo, e in alcuni casi anche economico-gestionale, dell'intero progetto della serie. E spesso è proprio la figura dello showrunner a incarnare l'oggetto di culto dei fan di una serie, o a essere il principale polo di attrazione e di creazione di attese da parte del pubblico per un particolare prodotto. Il ruolo extratestuale dell'autore di culto è in larga parte creato dai fans stessi che attribuiscono a una specifica figura l'aura della creatività assoluta, ma dal punto di vista produttivo scegliere una figura autoriale già oggetto di culto evidenzia una scelta o un orientamento verso un target di pubblico specifico, si cerca di costruire una serie di culto. Possiamo dire che, per assurdo, è proprio in una forma narrativa come la serie televisiva, la cui produzione è quanto di più distante dalla centralità della figura di un autore unico si possa pensare, che sembra avvenire il recupero della funzione-autore. Ma questo non a caso avviene in prodotti televisivi in cui si cerca di ottenere un effetto particolare di fidelizzazione da parte del pubblico, appunto nelle cult series (Hills 2002: 133).

\subsection{Narrazione aperta}

Il secondo meccanismo generatore di culto nelle serie tv, identificato da Hills, consiste in quella che chiama l'apertura indefinita della narrazione ("endlessly deferred narration"). Il concetto fa riferimento a quella che è la principale peculiarità strutturale della serialità, vale a dire la lunga durata. La serializzazione del racconto, il suo prolungarsi o dilatarsi potenzialmente all'infinito, è l'aspetto che lascia spazio a un particolare meccanismo generatore di culto tutto interno al testo. Si genera il culto nelle serie tv, ma questo vale anche per le saghe cinematografiche, quando l'attenzione dello spettatore viene concentrata lungo l'intero arco narrativo su una singola questione o 


\section{Ocula}

Vol 21, No 22 (April 2020) • DOI: 10.12977/ocula2020-16

Andrea Bernardelli | Serie tv di culto, ovvero il culto delle serie tv. Protagonisti seriali come cult icons nell'epoca della complex tv

quesito centrale, o anche su un insieme di questioni, ma comunque tra loro strettamente correlate (Hills 2002: 134).

In questo caso viene in mente il ruolo svolto da quello che viene chiamato l'arco narrativo stagionale, o a volte anche inter-stagionale (non a caso in inglese detto myth arc), in particolare in serie complesse come Lost o Il trono di spade. Quello che viene definito come arco narrativo non è altro che una domanda o questione centrale che regge il peso dell'intera struttura narrativa; nel caso di Lost la questione da risolvere è rappresentata dal mistero stesso dell'isola, nel Trono di spade è il "chi alla fine avrà il potere assoluto", appunto il trono del titolo.

L'esempio dato da Hills è quello della pluridecennale serie televisiva Doctor Who in cui, fa notare, il titolo è già di fatto "la questione" che tiene insieme la serie e le sue diverse stagioni, e di conseguenza la curiosità dei suoi fans. È il protagonista stesso in questo caso a essere il mistero che mantiene attiva la "endlessly deferred narration". Come dice Hills,

Threatened with narrative exhaustion, the programme struggled to find a way to 'regenerate' the sense of mystery which it had carried since its inception. Doctor Who projects its endlessly deferred narrative almost entirely upon the (non-) identity of its (anti-) hero: other cult series have displayed similar tendencies with regards to particular characters. (Hills 2002: 135)

In Star Trek, altra celebre cult series, è il personaggio del "signor Spock" a diventare uno dei punti di domanda che reggono la continuità dell'arco narrativo, in particolare è la sua differenza, o identità a seconda dei punti di vista, con l'umano a essere oggetto di discussione, essendo l'unico non-umano a bordo della Enterprise. Non a caso Spock è uno dei personaggi al centro dei discorsi e delle discussioni dei fans, i Trekkies, e vedremo come questo aspetto si intrecci con il terzo meccanismo di produzione di fan cult, la hyperdiegesis, di cui parleremo a seguire.

In sostanza quello che qui dice Hills porta a identificare un altro meccanismo, ancillare rispetto a questo, vale a dire il fatto che la serialità permette uno sviluppo della figura del personaggio lenta e approfondita nel corso degli episodi e delle stagioni. Potremmo a questo proposito citare l'esempio significativo del personaggio Gregory House, protagonista della serie House M.D. (2004-12). Il mistero della caratterizzazione e della identità del protagonista diventa sempre più centrale stagione dopo stagione, il che è evidente anche attraverso la sempre maggiore continuità narrativa data dalla serializzazione degli episodi. A partire dalla terza stagione gli sceneggiatori danno sempre più spazio alle linee narrative che riguardano House e ai suoi rapporti personali, mettendo in second'ordine i casi clinici di puntata che di volta in volta House e il suo team devono risolvere. ${ }^{2}$ La serializzazione di una serie nata come una

2 È in questa stagione che viene anche inserito l'elemento di continuità narrativa interepisodica dato dallo scontro di House con un opponente, rappresentato dal detective Michael Tritter. 


\section{Ocula}

Vol 21, No 22 (April 2020) • DOI: 10.12977/ocula2020-16

Andrea Bernardelli | Serie tv di culto, ovvero il culto delle serie tv. Protagonisti seriali come cult icons nell'epoca della complex tv

più tradizionale serie episodica - con segmenti narrativi più o meno autoconclusivi - è legata ad una sempre più forte centralità della "questione House", alla lenta costruzione di una sempre più complessa identità del protagonista. Da cui il culto concentrato sulla figura di House, fino a portare un ampio fandom a isolare frasi ed espressioni del personaggio e a definirli housemismi (housemisms), come nel caso del celebre "Preferisce un medico che le tenga la mano mentre muore, o uno che la ignori mentre cerca di guarirla?”.

$\mathrm{Nel}$ caso di questa serie nel creare le condizioni per il fan cult svolge un ruolo rilevante anche la forte caratterizzazione intertestuale e citatoria della narrazione stessa. La serie cita, di fatto in termini parodici, il genere medical più tradizionale,$^{3}$ ma soprattutto è legata nella struttura logica della narrazione alla forma del procedural investigativo. House M.D. è strutturato come un whodunnit, ha maggiori similarità con un poliziesco o un legal che con l'approccio più sentimentale del genere medical a cui dovrebbe appartenere per ambientazione. ${ }^{4}$ Gli aspetti drama della serie sono invece tutti orientati non sui casi clinici che di volta in volta, in maniera fredda e razionale, House risolve, ma sulla sua storia e identità personale. La logica investigativa soggiacente è legata anche a un altro aspetto rilevante della serie. Per dichiarazione esplicita degli autori il personaggio di Gregory House era stato modellato sulla figura dell'investigatore Sherlock Holmes, un personaggio transmediale già oggetto di un vasto culto.

Anche in questo caso l'intertestualità legata alla figura del personaggio House porta verso la costruzione del culto. Le frasi isolabili e citabili ("sgangherabili" avrebbe detto Eco), l'intreccio di diversi generi narrativi e la loro citazione parodica o meno, il riferimento a un'altra cult icon come Sherlock, tutto questo induce alla generazione di un fandom e di un culto dell'oggetto narrativo "House".

Questa importanza del ruolo svolto dalla figura complessa del personaggio - non così evidenziata da Hills come avrebbe dovuto - è invece al centro dell'attenzione di Roberta Pearson (2003: 6). È evidente come il personaggio protagonista delle cult series sia quello che manifesta possibilità narrative il più possibile aperte. Si tratta spesso di un personaggio che sta subendo un percorso di formazione, che sta cambiando o è in divenire. Questo genera una narrazione fatta di potenzialità, di percorsi alternativi per il personaggio, e quindi la migliore forma di endlessly deferred narrative.

Importante il fatto che il crollo o la chiusura del processo della endlessly deferred narrative segni la crisi del culto. Hills porta ad esempio il caso della serie Moonlighting (1985-89) in cui, una volta portata a una risoluzione la tensione sessuale esistente tra i due co-protagonisti - interpretati da Cybill Shepherd e Bruce Willis, colleghi nell'agenzia investigativa Blue Moon e che

3 La figura di House è il rovesciamento del medico buono e sentimentale alla Dottor Kildare. House è un accanito spettatore, un fan, del medical drama General Hospital che viene citato spesso nella serie in modo ironico.

4 Lo stesso ideatore della serie, David Shore, dichiarava di avere l'intenzione "di realizzare una serie poliziesca ambientata nel mondo medico in cui un gruppo di medici cercavano di diagnosticare l’indiagnosticabile”. 


\section{Ocula}

Vol 21, No 22 (April 2020) • DOI: 10.12977/ocula2020-16

Andrea Bernardelli | Serie tv di culto, ovvero il culto delle serie tv. Protagonisti seriali come cult icons nell'epoca della complex tv

costantemente cercano di negare attraverso l'ironia la loro reciproca attrazione -, la narrazione perde la propria ragion d'essere per gli allora numerosi fans della serie (Hills 2002: 135). Allo stesso modo nella serie Twin Peaks, basata sulla domanda fondamentale "Who killed Laura Palmer", una volta rivelata l'identità di "Bob" avviene un collasso ermeneutico, la serie non riesce più a trovare un focus per gli spettatori.

Quel che importa a questo punto è di aver potuto evidenziare che queste strutture narrative aperte e continue, basate su un quesito centrale la cui soluzione è continuamente procrastinata, sono create consapevolmente, vengono costruite in questo modo per determinare un particolare rapporto con gli spettatori, una relazione di fidelizzazione estremamente forte e che può generare, insieme ad altri fattori, il culto mediale. Importante sembra essere il ruolo svolto dall'inserimento del singolo testo (la serie tv) nella rete dei culti mediali già esistenti. Vale a dire che è fondamentale nel meccanismo della cult tv la presenza di richiami intertestuali, come sottolineava già Eco. Senza dimenticare che quando questi richiami sono troppo smaccati, evidenti, o forzati si può ottenere l'effetto contrario, riducendo le potenzialità di oggetto di culto della serie. Le competenze intertestuali dello spettatore, in particolare del fan, devono essere stimolate in modo raffinato e ricercato, perché devono supportare il commento e la discussione mai banale del fandom. Ma di questo parleremo a proposito della hyperdiegesis.

\subsection{Metatesti complessi}

La cult series deve offrire la possibilità di costruire diegesi complesse e estese, anche al di là dei confini della narrazione stessa (hyperdiegesis). Questo vuole dire che una serie di culto deve avere la potenzialità di creare un vasto e dettagliato spazio narrativo, ma anche di commento e di critica, di cui solo una parte è di fatto "nel testo". Ad esempio, i fans di Star Trek collegano aspetti della serie tv con eventi, personaggi e particolari dei film legati al medesimo mondo narrativo. I fans in questo modo è come se costruissero un mondo narrativo esteso, come il Marvel Cinematic Universe, ma dal basso. La costruzione di un universo narrativo di Star Trek è quindi resa coerente dagli stessi spettatori esperti, anche quando questa coerenza non esisteva.

Questo meccanismo metatestuale presuppone la possibilità di prendere visione delle serie tv in maniera critica, in sostanza di poterle "rivedere" in maniera analitica. Mentre in precedenza questo era reso possibile solo attraverso le repliche, poi accresciuta dai canali dedicati e dall'uso di DVD, oggi le serie tv sono prodotti con una vita commerciale molto più lunga, in particolare attraverso lo streaming, o le OTT, anche senza più una vera e propria "messa in onda". Ora l'attenzione critica dello spettatore, e del fan in particolare che si avvicina alla maniacalità, può essere esaudita. Oltre a questo esiste oggi una maggiore possibilità di fruire di tutti quegli apparati genericamente definibili come paratestuali, sia ufficiali (recensioni, lanci, preview, trailes, commenti giornalistici) che non (blog, fanfiction, siti di fandom, social media in genere). Tutto questo aumenta le possibilità da parte del fan di serie tv di accrescere 


\section{Ocula}

Vol 21, No 22 (April 2020) • DOI: 10.12977/ocula2020-16

Andrea Bernardelli | Serie tv di culto, ovvero il culto delle serie tv. Protagonisti seriali come cult icons nell'epoca della complex tv

la propria competenza e conoscenza della serie, e quindi di potere a sua volta creare propri apparati metatestuali o paratestuali che dir si voglia. In un certo senso ci troviamo di fronte sempre più a un fan di qualità, non più un semplice entusiasta, ma quello che lo stesso Hills ha identificato come il fan-scholar (2002: 16), il fan dotato di una capacità critica pari a quella di un accademico anche senza esserlo istituzionalmente (diverso quindi, in un certo senso speculare, rispetto allo scholar-fan o aca-fan sempre di Hills, termine ripreso poi da Jenkins). ${ }^{5}$ Daniela Cardini ha identificato una figura simile definendo il tele-cinefilo (Cardini 2014). Si tratta di spettatori che amano il cinema e ne possiedono una certa competenza e che arrivano a interessarsi alla serialità televisiva perché le riconoscono virtù cinematografiche. Sono esperti di audiovisivi, non semplici spettatori presi dall'entusiasmo per un racconto o per un attore.

Pearson (2003: 6) nota come sia fondamentale il fatto che le cult tv series generino un metatesto complesso e articolato in cui i fans trovino il piacere della sfida intellettuale, rappresentata dalla sua discussione e articolazione dialogica. Il ruolo svolto dai social networks è in questo caso fondamentale, sia nel divulgare il commento esteso dei fan, sia nel definire anche il livello della discussione, grazie al fatto che sono gli stessi partecipanti a selezionarsi per livelli di competenza. In sostanza il metatesto critico sulle serie tv non è solo la prova del culto a esse riservato o meno, - e la loro estensione quantitativa prova del loro essere culto più o meno rilevante -, ma è anche lo spazio in cui avviene il passaggio di quel metatesto in paratesto, più o meno ufficiale. Il commento in rete dei fandom diventa sempre più parte del testo stesso della serie, lo spazio testuale utile per comprenderne il senso e spesso indispensabile per farne una analisi critica.

\section{Il culto del personaggio "difficile"}

Torniamo ora sull'importanza del ruolo svolto dalla figura del personaggio protagonista nel definire l'effetto fidelizzante sullo spettatore legato alla endlessly deferred narrative. In particolare concentreremo il nostro interesse sui protagonisti delle cosiddette serie tv "complesse".

Abbiamo visto come il personaggio per diventare potenziale oggetto di culto deve essere per lo spettatore un puzzle, deve costituire una serie di domande irrisolte, o la cui soluzione sia continuamente procrastinata. Lo spettatore deve essere portato a seguire la costruzione e/o la trasformazione di puntata in puntata del personaggio protagonista, deve "vivere" la costruzione della complessità di quella figura narrativa. Un personaggio che agli occhi dello spettatore incarna le caratteristiche di un tipo, così come inteso da Eco, deve rinviare l'attenzione a temi e questioni che portino a quella discussione che caratterizza il forensic fan o il fan-scholar. In questo processo di costruzione della complessità del personaggio un ruolo non indifferente viene svolto, come

5 Si tratta di quella figura di spettatore attivo e narratologicamente informato che secondo Mittell costituisce il forensic fandom (2015: 52). 


\section{Ocula ${ }^{22}$}

Vol 21, No 22 (April 2020) • DOI: 10.12977/ocula2020-16

Andrea Bernardelli | Serie tv di culto, ovvero il culto delle serie tv. Protagonisti seriali come cult icons nell'epoca della complex tv

detto, dai richiami intertestuali e extratestuali che quel personaggio porta con sé. Stiamo parlando quindi in sostanza di un personaggio aperto, dotato di potenzialità interpretative infinite per lo spettatore, e non di un personaggio topos o chiuso.

Per ottenere una figura di personaggio che permetta una forte apertura, che rinvii a una serie di questioni aperte indefinitamente, è inevitabile lavorare sulla costruzione di un personaggio articolato, di difficile comprensione, in sostanza complesso. Ma per trasformarlo in cult icon si deve fare in modo che il suo mistero resti irrisolto, che potenzialmente quel personaggio possa fornire ancora materiale alla discussione e a virtuali seguiti indefinitamente. Come nel mito classico, il protagonista antieroico contemporaneo deve fornire con continuità stimoli per comprendere la realtà in cui viviamo. Ma nel nostro regime mediale può fare questo solo trasformandosi in un nuovo tipo di mito, un mito a bassa intensità.

Ortoleva (2019: xv) ha coniato questa espressione per identificare le caratteristiche dei nuovi miti contemporanei, vale dire delle diverse narrazioni mediali che circolano nella cultura occidentale. ${ }^{6}$ Le mitologie classiche, o pre-moderne, sono quelle definite da Ortoleva ad alta intensità, vale a dire costituite da "narrazioni sacrali e circondate da cerimoniali". I tratti essenziali di questa miticità, quella greco-romana e delle popolazioni extraeuropee di interesse etnografico, sarebbero: 1. la distanza temporale in cui avvengono gli eventi narrati rispetto al mondo del narratore e dell'ascoltatore; 2. lo stretto rapporto dei racconti con un sistema di obblighi e divieti legati a un cerimoniale; 3. la presenza di figure diverse dagli esseri umani, collocati in un mondo superiore o infero, vale a dire divinità, demoni, animali parlanti, in sostanza entità soprannaturali. I miti contemporanei a bassa intensità sono invece caratterizzati: 1. dal fatto di collocare il racconto nel nostro tempo e in un mondo riconoscibile e reale; 2 . sono narrazioni oggetto di consumo, libero e personale, in sostanza si ascoltano i miti a bassa intensità per libera scelta, non per obbligo o seguendo tempi cerimoniali; 3. i protagonisti di questi racconti sono esseri umani, uguali a noi. Ovviamente i due modi, o intensità narrative, del mito non sono storicamente esclusivi; continuano di fatto a convivere e a intrecciarsi l'uno con l'altro anche nella contemporaneità.

Uno dei miti mediali contemporanei a bassa intensità individuato da Ortoleva è quello del criminale o del gangster. La figura del criminale ha assunto una dimensione mitica, e quindi culturalmente centrale e fondativa, grazie a tre principali sue caratteristiche. Prima di tutto il criminale rappresenta, come in ogni struttura mitologica che si rispetti, il lato ctonio o oscuro della narrazione mitologica mass mediale a bassa intensità. Ma se il criminale è l'eroe negativo, così come Lucifero è l'angelo del male, nella logica della bassa intensità non è più una figura immortale, ha dei limiti umani e la sua fine è spesso tragica. Inoltre il gangster rappresenta quella che Ortoleva definisce

6 Lo stesso Jenkins (2007: 116 e 164) definiva le storie e i racconti che circolano nei mass media come "contemporary myths", senza però approfondire il discorso ulteriormente. 


\section{Ocula}

Vol 21, No 22 (April 2020) • DOI: 10.12977/ocula2020-16

Andrea Bernardelli | Serie tv di culto, ovvero il culto delle serie tv. Protagonisti seriali come cult icons nell'epoca della complex tv

come "una delle controcorrenti" rispetto alla cultura dominante, espressione narrativa delle contraddizioni della società in cui viviamo. È il risvolto tragico di una cultura di massa che invita all'ottimismo e che, attraverso la figura del criminale con la sua negatività, ne svela l'inganno. Infine il ruolo del cattivo è non solo fondamentale nella stessa struttura della narrazione mitica, ma fondativo: senza un villain non si potrebbe avere una storia.

La figura del criminale è inoltre frutto della sintesi di opposti che rappresentano ogni tipo di ambiguità culturale: il gangster è colui che ammiriamo, per il suo successo e il suo potere, ma che allo stesso tempo temiamo, il bene e il male non sono mai così nettamente separati.7 Spesso la sua rappresentazione serve anche a mettere in discussione le gerarchie sociali: l'alto e il basso si confondono, e l'aspirazione del criminale al "bel mondo" spesso è ciò che lo porta a una tragica conclusione. Il criminale nella mitologia contemporanea è spinto da una irrefrenabile ambizione verso il potere. Questa sua ambizione è simile alla hybris classica e non può che condurlo, come notava Warshow, a un tragico destino e alla punizione, perché ha avuto la pretesa di emergere dalla massa anonima senza accontentarsi di una "felicità media". ${ }^{8}$

La figura tragica e ribelle, in un certo senso prometeica, del criminale, delineata da Ortoleva come mito mediale contemporaneo, seppure a bassa intensità, ha un'altra definizione, che è quella dell'antieroe. Nella serialità televisiva contemporanea emerge come elemento fondamentale per la costruzione di una struttura narrativa complessa di alcuni prodotti la presenza di una particolare caratterizzazione del protagonista, quella di un personaggio negativo, moralmente discutibile, in un certo senso "sbagliato", vale a dire di una figura anti-eroica (Mittell 2015: 142). La principale questione che è stata posta riguardava il motivo per cui come spettatori ci appassioniamo alle vicende di personaggi di questo genere, risolta attraverso l'individuazione di una serie di dispositivi narrativi utili ad attenuare il peso dell'immoralità o inadeguatezza di questi personaggi. ${ }^{9}$ Ma esiste un'altra domanda da porsi: quale funzione rivestono questi personaggi antieroici nella nostra cultura? A cosa serve la loro rappresentazione, a quali esigenze culturali risponde?

7 Robert Warshow, ispiratore delle riflessioni di Ortoleva, scriveva nel 1948 nel suo saggio "The Gangster as Tragic Hero", a proposito del ruolo della figura del criminale nell'immaginario popolare: "The real city, one might say, produces only criminals; the imaginary city produces the gangster: he is what we want to be and what we are afraid we may become» (Warshow 2001: 101).

8 "At bottom, the gangster is doomed because he is under the obligation to succed, not because the means he employs are unlawful. In the deeper layers of the modern cosciousness, all means are unlawful, every attempt to succeed is an act of aggression, leaving one alone and guilty and defenseless among enemies: one is punished for success. This our intolerable dilemma: that failure is a kind of death and success is evil and dangerous, is - ultimately - impossible. The effect of the gangster film is to embody this dilemma in the person of the gangster and resolve it by his death. The dilemma is resolved because it is his death, not ours. We are safe; for the moment, we acquiesce in our failure, we can choose to fail» (Warshow 2001: 103).

9 Per una sintesi della questione, e per l'ampia bibliografia a riguardo, vedi Bernardelli (2016). 


\section{Ocula}

Vol 21, No 22 (April 2020) • DOI: 10.12977/ocula2020-16

Andrea Bernardelli | Serie tv di culto, ovvero il culto delle serie tv. Protagonisti seriali come cult icons nell'epoca della complex tv

Come dice Mittell, il protagonista complesso - perché antieroico e quindi dotato di ambiguità e molteplici possibilità interpretative - è essenziale per sostenere una trama narrativa altrettanto complessa. Ma questa è una spiegazione tautologica che non risponde al quesito riguardo al successo di questo tipo di personaggio, e della relativa modalità narrativa. L'antieroe, il criminale, il gangster, sono figure che generano culto, attrazione nel pubblico, e non solo perché mediante qualche abile trucco narrativo si riesce ad attenuarne la negatività, ci deve essere altro. Abbiamo visto, attraverso Eco e Ortoleva in particolare, come sia possibile delineare l'identità potenziale di un personaggio che potremmo definire come predestinato al culto (e che con sé trascina la narrazione stessa in cui è inserito). A questo punto però dobbiamo cercare di capirne il funzionamento attraverso alcune esemplificazioni di questo tipo di personaggio nella serialità televisiva contemporanea.

Possiamo partire da quello che viene ritenuto il capostipite dei bad guys della serialità televisiva contemporanea - o quantomeno il più rappresentativo -, Tony Soprano, il protagonista della serie tv The Sopranos (HBO, 19992007)..$^{10}$ Perché possiamo considerarlo un personaggio aperto, da cui quindi si possa generare un culto da mito a bassa intensità? Tony è a capo di una famiglia mafiosa del New Jersey e vive l'ambiguità di dover essere anche un bravo padre di famiglia. Noi spettatori seguiamo la difficile esistenza di questo personaggio attraverso i compromessi che un gangster deve accettare per far convivere la propria identità criminale con la sua "normale" identità quotidiana; conflitti con la moglie, con i figli, e con una dispotica madre (non estranea alla gestione dell'altra "famiglia", quella criminale). Tanto che Tony, nonostante sia una cosa decisamente in contrasto con l'etica dell'omertà mafiosa, deve rivolgersi, per cercare sollievo dai suoi attacchi d'ansia, a una psicanalista. Ma dove troviamo quel meccanismo di apertura del personaggio che lo rende un mistero irrisolto, che lo può portare sulla strada della cult icon?

Tony di fatto non cambia "all'esterno" - la sua identità sarà sempre la stessa, è un gangster e tale rimarrà fino alla fine dell'ultima stagione -, ma cambia "dentro" per noi spettatori che ne conosciamo i moti interiori. In questo senso riveste un ruolo fondamentale la figura della psicanalista perché è attraverso i dialoghi delle sedute che noi spettatori veniamo a conoscere le questioni irrisolte della personalità di Tony. È l'interiorità di Tony Soprano a rappresentare il mistero, o la catena di questioni, che tengono sempre attiva l'attenzione dello spettatore, e aperta la possibilità interpretativa del personaggio. Per lo spettatore Tony rimane un mistero, un insieme di interrogativi, visto che dovrà continuare a domandarsi in che modo quel personaggio potrà sopportare il peso e le pressioni della propria esistenza. Di conseguenza, quello che prova il personaggio è quanto potremmo provare noi stessi di fronte alle difficoltà quotidiane; non è una umanizzazione del criminale, ma è nel criminale che viene rappresentato l'umano. Da cui la possibilità di individuare la caratterizzazione di questa narrazione come un mito a bassa intensità. Il protagonista di questa narrazione è umano, così come dice Ortoleva,

10 Per una panoramica sulle analisi della serie v. Lavery, Howard e Levinson (2011). 


\section{Ocula ${ }^{22}$}

Vol 21, No 22 (April 2020) • DOI: 10.12977/ocula2020-16

Andrea Bernardelli | Serie tv di culto, ovvero il culto delle serie tv. Protagonisti seriali come cult icons nell'epoca della complex tv

e allo stesso tempo è tipico in quanto richiama valori e questioni universali, nel senso di Eco.

La figura di Tony Soprano inoltre è un vero e proprio snodo intertestuale. Il suo personaggio evoca l'intera tradizione dei gangster movies, ma soprattutto la filmografia incentrata sulle famiglie mafiose italo-americane, di cui è in un certo senso punto di riferimento Il Padrino. Il fatto che questo insieme di riferimenti intertestuali abbia avuto delle forti ricadute sul più ampio contesto culturale e ideologico lo dimostra l'ondata di polemiche suscitata dalla serie, in particolare da parte delle comunità italo-americane che si sono sentite offese dalla rappresentazione stereotipata della loro identità culturale come quella di criminali (v. Gardaphé 2016). In sostanza la figura di Tony Soprano ha un tale potere tipico da creare da un lato una polemica culturale e, dall'altro, un culto mediatico (v. Monaco 2007, Couldry 2007).

Un discorso simile è possibile farlo per il protagonista di Breaking Bad, Walter White, altra cult icon del mondo delle serie tv contemporanee. ${ }^{11} \mathrm{Ma}$ in questo caso il meccanismo della endlessly deferred narrative sul personaggio sembra essere opposto a quello indicato per Tony Soprano. Se Tony in superficie non cambiava, ma era al suo interno che subiva continue trasformazioni ed evoluzioni, nel caso di Walter White assistiamo alla trasformazione esterna del personaggio da onesto e succube insegnante di chimica a feroce signore della droga. Ma in realtà, come ci viene fatto lentamente capire nel corso della serie, nella sua interiorità questa figura non è mai cambiata. Il male, o la negatività, Walter l'aveva dentro di sé fin da principio. Non è stato lo scontro con la realtà ad averlo trasformato, quelle situazioni hanno fatto solo emergere il male che era in lui. La tipicità del personaggio consiste nel fatto che lo stesso meccanismo possiamo pensarlo come potenzialmente attivo in ognuno di noi. Come diceva Warshow «he is what we want to be and what we are afraid we may become» (2001: 101). Il personaggio Walter White è tipico e umano, ha quindi in sé qualcosa di iconico, da mito a bassa intensità. Possiamo aggiungere anche in questo caso che la sua figura evoca una serie di riferimenti intertestuali letterari e filosofici di ampia portata legati alla dialettica morale tra il bene e il male: da Caino e Abele passando poi per Dr. Jekyll e Mr. Hyde, le rappresentazioni narrative del dualismo tra bene e male sono innumerevoli, tutte in un modo o nell'altro evocate da quanto accade a Walter White/Heisenberg (v. anche Dusi 2016).

Possiamo trovare un'altra esemplificazione di un personaggio diventato cult icon, questa volta nel contesto mediale italiano; si tratta della figura di Ciro Di Marzio, detto anche "l'Immortale", di Gomorra. La serie (Sky, 2014in produzione). ${ }^{12}$ Ispirato alla reale figura del camorrista Gennaro Marino, detto Genny o'McKay esponente del clan degli scissionisti di Secondigliano, è diventato un personaggio punto di riferimento per i fans della serie, nonostante di fatto la narrazione sia stata costruita secondo una struttura corale,

11 Sulla serie e sul suo fandom v. Checcaglini (2014), Pierson (2014), Dusi (2016).

12 Vedi Napoli, Tirino (2015); per una visione sociologica "colpevolista" del fandom di Gomorra, vedi Salzano (2018). 


\section{Ocula}

Vol 21, No 22 (April 2020) • DOI: 10.12977/ocula2020-16

Andrea Bernardelli | Serie tv di culto, ovvero il culto delle serie tv. Protagonisti seriali come cult icons nell'epoca della complex tv

con diversi personaggi emergenti o di rilievo (tra gli altri anche il personaggio di Gennaro "Jenny" Savastano, con cui la figura di Ciro ha tenuto attivo un dualismo narrativo per ben tre stagioni della serie). Le frasi e le situazioni narrative che coinvolgono questo personaggio sono citate e ricordate dai fans, tanto da essere stato al centro della polemica che ha coinvolto nel suo insieme questa serie e i suoi supposti effetti sui suoi giovani spettatori. ${ }^{13}$ Lo status di cult icon anche in questo caso è indotto da una costruzione della figura del personaggio non solo "tonda" e aperta alle interpretazioni degli spettatori, ma anche per la sua funzione di snodo intertestuale ed extratestuale. In Gomorra l'impatto dell'eccesso di realismo delle situazioni narrate viene attenuato agli occhi dello spettatore da una recitazione fortemente teatrale o finzionale. Ma proprio questa recitazione melodrammatica "da sceneggiata napoletana" porta alla possibilità di isolare situazioni narrative che diventino iconiche, o cariche di significati extratestuali, per lo spettatore. Per assurdo, in questo caso, è l'eccesso di finzionalizzazione a determinare la risposta extrafinzionale. L'iperrealismo della finzionalità nella recitazione porta a quella che Eco aveva definito come sgangherabilità: situazioni apparentemente realistiche nel loro rinvio extratestuale alla cronaca, diventano così isolabili perché suonano "finte". Diventano citabili proprio perché in tal modo perdono il loro eccessivo peso morale ed emotivo se direttamente correlati alla cruda realtà degli eventi evocati. Diventano simboli finzionali di un qualcosa troppo reale da potere essere sopportato senza questo filtro narrativo. Il ruolo di cult icon della figura di Ciro deriva dal suo essere un camorrista palesemente finzionale - ed è qui che può entrare in gioco ed essere sopportabile il suo status di anti-eroe -, ma che rinvia a qualcosa che finzionale non è, e che sarebbe insopportabilmente inquietante per lo spettatore senza il filtro della figura iconica dell'antieroe. Come ha detto Saviano (2014), per rispondere alle polemiche sulla serie, lui non intendeva raccontare la camorra al mondo, ma il mondo attraverso la camorra. In sostanza la narrazione della camorra nella serie sta per qualcos'altro, è tipica nel senso di Eco, e rinvia alle logiche latamente criminali su cui il mondo contemporaneo vive.

\section{Bibliografia}

Abbott, Stacey (ed.)

2010 The Cult Tv Book, London-New York, I.B. Tauris.

Bernardelli, Andrea

2016 Cattivi seriali, Roma, Carocci.

Boitani, Piero

1992 L'ombra di Ulisse. Figure di un mito, Bologna, il Mulino.

13 Interessante la risposta narrativa, e ironica, a queste polemiche data da The Jackal in una serie di cortometraggi intitolati Gli effetti di Gomorra sulla gente (< https://www.youtube.com/watch?reload=9\&v=xbWw8766ftI $>$ ). 


\section{Ocula ${ }^{22}$}

Vol 21, No 22 (April 2020) • DOI: 10.12977/ocula2020-16

Andrea Bernardelli | Serie tv di culto, ovvero il culto delle serie tv. Protagonisti seriali come cult icons nell'epoca della complex tv

Calabrese, Stefano; Conti, Valentina

2019 Che cos'è una fanfiction, Roma, Carocci.

Cardini, Daniela

2014 "Il tele-cinefilo. Il nuovo spettatore della Grande Serialità televisiva", in Tecnologia, immaginazione e forme del narrare, a cura di L. Esposito, E. Piga, A. Ruggiero, Between, IV.8, 2014, <http://www.betweenjournal.it>.

Checcaglini, Chiara

2013 "Created by. L'autore-star nella serialità televisiva contemporanea", in Maio (2013a), pp. 86-94.

2014 Breaking bad. La chimica del male: storia, temi, stile, Milano-Udine, Mimesis.

Couldry, Nick

2007 "On the Set of The Sopranos: Inside a Fan's Construction of Nearness", in Gray, Sandvoss, Harrington (2007), pp. 139-147.

Denson, Shane and Mayer, Ruth

2018 "Border Crossings: Serial Figures and the Evolution of Media", NECSUS. European Journal of Media Studies 7 (2), 65-84, 2018. DOI: <http://dx.doi. org/10.25969/mediarep/3460>.

Duffett, Mark

2013 Understanding Fandom. An Introduction to the Study of Media Fan Culture, New York, Bloomsbury Academic.

Dusi, Nicola

2016 "Intertestuale, intermediale e crossmediale, e il gusto dell'inatteso, in Breaking Bad", in A. Bernardelli, E. Federici, G. Rossini (a cura di), Between, VI.11, "Forme, strategie e mutazioni del racconto seriale", <http://www. betweenjournal.it>.

Eco, Umberto

1964 "Il mito di Superman" (1962), in Id., Apocalittici e integrati. Comunicazioni di massa e teorie della cultura di massa, Milano, Bompiani, pp. 219-61.

1964a "L'uso pratico del personaggio", in Id., Apocalittici e integrati. Comunicazioni di massa e teorie della cultura di massa, Milano, Bompiani, pp. 187-218.

1977 “Casablanca, o la rinascita degli dei” (1975), in Id., Dalla periferia dellimpero. Cronache di un nuovo medioevo, Milano, Bompiani, pp. 138-143.

1985 "L’innovazione nel seriale", in U. Eco, Sugli specchi e altri saggi, Milano, Bompiani, 1985, pp. 125-146).

Gardaphé, Fred

2016 "Running Joke: Criticism of Italian American Culture through Comedy in The Sopranos", in A. Bernardelli, E. Federici, G. Rossini (a cura di), Between, VI.11, "Forme, strategie e mutazioni del racconto seriale", <http://www. betweenjournal.it>.

Gwenllian-Jones, Sara and Pearson, Roberta E. (Eds.)

2004 Cult Television, Minneapolis, University of Minnesota Press.

Gray, Jonathan Alan; Sandvoss, Cornel; Harrington, C. Lee (edited by)

2007 Fandom: Identities and Communities in a Mediated World, New York, New York University Press. 


\section{Ocula ${ }^{22}$}

Vol 21, No 22 (April 2020) • DOI: 10.12977/ocula2020-16

Andrea Bernardelli | Serie tv di culto, ovvero il culto delle serie tv. Protagonisti seriali come cult icons nell'epoca della complex tv

Hills, Matt

2002 Fan Culture, London-New York, Routledge.

Jenkins, Henry

2006 Convergence Culture, New York, The New York University Press (tr. it. Cultura convergente, Milano, Apogeo, 2014).

2007 Fans, Bloggers and Gamers, New York, The New York University Press (tr. it. Fans, bloggers e videogamers. L'emergere delle culture partecipative nell'era digitale, Milano, Franco Angeli, 2008).

Lavery, David; Howard, Douglas L.; Levinson, Paul (edited by)

2011 The Essential Sopranos Reader, Lexington, University Press of Kentucky.

Maio, Barbara (a cura di)

2013 Cult Television, Roma, Rigel.

2013a Media Stardom. Fama, successo e gossip tra passato e futuro, Roma, Rigel.

Mittell, Jason

2015 Complex Tv, New York-London, New York University Press, 2015 (tr. it. Complex tv. Teoria e tecnica dello storytelling delle serie tv, Roma, minimum fax, 2017)

Monaco, Jeannette

2007 Performing Fandom, Performing Community: A Case Study of The Sopranos and Its Online Fandom, PhD Thesis, University of Bristol, <https://ethos. bl.uk/OrderDetails.do?uin=uk.bl.ethos.504528>.

Monteleone, Franco

2005 Cult series. Le grandi narrazioni televisive nell'America di fine secolo, 2 voll., Roma, Dino Audino.

Napoli, Antonella; Tirino, Mario

2015 "Gomorra remixed. Transmedia storytelling tra politiche di engagement mainstream e produttività del fandom”, Series. International journal of Serial Narratives, I, 2, Winter 2015, pp. 193-206.

Ortoleva, Peppino

2019 Miti a bassa densità. Racconti, media, vita quotidiana, Torino, Einaudi.

Pearson, Roberta E.

2003 "Kings of Infinite Space: Cult Television Characters and Narrative Possibilities", Nottingham, Nottingham University Papers, <https://www. nottingham.ac.uk/scope/documents/2003/november-2003/pearson.pdf>.

Pierson, David P. (edited by)

2014 Breaking Bad: Critical Essays on the Contexts, Politics, Style, and Reception of the Television Series, Lanham, Lexington Books.

Salzano, Diana

2018 “Gomorra. La serie. Transnazionalità di un'epica senza eroi”, in D. Salzano, I.S. Germano, F. Ferzetti, Bologna (a cura di), Sociologie del mutamento II, Esculapio, pp. 313-330.

Saviano, Roberto

2014 "Perché sono tutti cattivi nella Gomorra che va in tv", la Repubblica, 10 giugno 2014. 


\section{Ocula ${ }^{22}$}

Vol 21, No 22 (April 2020) • DOI: 10.12977/ocula2020-16

Andrea Bernardelli | Serie tv di culto, ovvero il culto delle serie tv. Protagonisti seriali come cult icons nell'epoca della complex tv

Scaglioni, Massimo

$2006 \mathrm{Tv}$ di culto. La serialità televisiva americana e il suo fandom, Milano, Vita $\mathrm{e}$ Pensiero.

Warshow, Robert

2001 The Immediate Experience. Movies, Comics, Theatre and Other Aspects of Popular Culture, Cambridge (Mass.) and London, Harvard University Press.

Williams, Rebecca

2015 Post-Object Fandom. Television, Identity, and Self-narrative, New YorkLondon, Bloomsbury Academic.

Andrea Bernardelli (Bologna, 1962) insegna Semiotica all'Università degli studi di Perugia e alla IULM di Milano. È autore dei volumi Che cos'è la narrazione (Roma, Carocci, 2019), Che cos'é una serie televisiva (con G. Grignaffini, Roma, Carocci, 2017), Cattivi seriali. Personaggi atipici nelle produzioni televisive contemporanee (Roma, Carocci, 2016), Semiotica. Storia, contesti e metodi (con E. Grillo, Roma, Carocci, 2014), Che cos'è l'intertestualità (Roma, Carocci, 2013), Il testo narrativo (con R. Ceserani, Bologna, il Mulino, 2005), Intertestualità (Firenze, La Nuova Italia, 2000), La narrazione (Roma-Bari, Laterza, 1999), Il parlato e lo scritto (con R. Pellerey, Milano, Bompiani, 1999). 